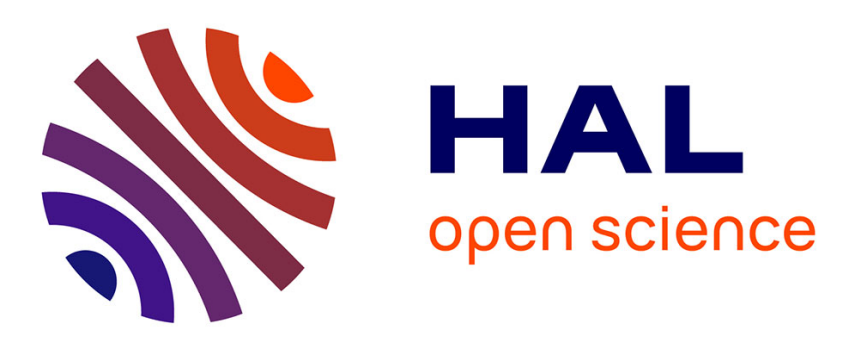

\title{
Prospection semi-probabiliste de l'arbre de ruine d'une structure existante
}

\author{
Franck Schoefs, Jean-François Dubé, Jean Goyet
}

\section{To cite this version:}

Franck Schoefs, Jean-François Dubé, Jean Goyet. Prospection semi-probabiliste de l'arbre de ruine d'une structure existante. Revue Française de Génie Civil , 2002, 6 (3), pp.435-446. 10.1080/12795119.2002.9692379 . hal-01007226

\section{HAL Id: hal-01007226 \\ https://hal.science/hal-01007226}

Submitted on 18 Mar 2017

HAL is a multi-disciplinary open access archive for the deposit and dissemination of scientific research documents, whether they are published or not. The documents may come from teaching and research institutions in France or abroad, or from public or private research centers.
L'archive ouverte pluridisciplinaire HAL, est destinée au dépôt et à la diffusion de documents scientifiques de niveau recherche, publiés ou non, émanant des établissements d'enseignement et de recherche français ou étrangers, des laboratoires publics ou privés. 


\title{
Prospection semi-probabiliste de l'arbre de ruine d'une structure existante
}

\section{Franck Schoefs* - Jean-François Dubé* - Jean Goyet**}

\author{
* Laboratoire de Génie Civil Nantes Saint Nazaire \\ Faculté des sciences, 44072 F- Nantes cedex 03 \\ \{Franck.schoefs; Jean.francois.dube\}@physique.univ-nantes.fr \\ ** Bureau Veritas, Division Marine, Département Recherche \\ F- 92077 Paris la Défense cedex \\ jean.goyet@bureauveritas.com
}

\begin{abstract}
RÉSUMÉ. Les grands ouvrages de génie civil conçus il y a plusieurs décennies font l'objet d'études particulièrement complexes de la part de l'opérateur en charge de leur maintenance et des organismes de certification. La notion d'intégrité structurelle est au cœur du processus d'évaluation de leur état. L'effet d'échelle des ouvrages et le vieillissement de leurs matériaux constitutifs imposent une approche probabiliste, tant au niveau de la modélisation que de l'estimation des paramètres. Le secteur offshore, confronté à cette problématique depuis le début des années 1990, appuie ses décisions sur l'analyse d'arbres de ruine. Cet article propose une alternative semi-probabiliste à la construction de tels arbres fondée habituellement soit sur des critères purement déterministes, soit sur une analyse en fiabilité de la structure.
\end{abstract}

ABSTRACT. Large civil engineering structures, designed few decades ago, are actually submitted to complex studies from operators and certification authorities. The concept of structural integrity is the aim of the process for structural assessment. Effects of structural scale, uncertainty on material properties lead to state the importance of a probabilistic approach for the modelling and the parameters estimation. Offshore sector is asked for the reassessment of structures since the beginning of the 90's and has developed to this aim several aid-tools. The most useful is the fault-tree built following a deterministic scheme or a reliability analysis of fault scenario. Since this computation are numerical codes dependent, complementary scheme are to be developed to give the most complete information on the structural integrity. This paper proposes an iterative building of fault-tree based on a semiprobabilistic analysis of the structural integrity.

MoTS-CLÉS: structures existantes, modélisation probabiliste, arbre de ruine, structures offshore.

KEYWORDS : existing structures, probabilistic modelling, fault trees, offshore platforms. 


\section{Introduction}

Au cours du vingtième siècle, l'amélioration des techniques de construction, des méthodes de conception et des matériaux du génie civil a permis de donner tout son sens à la durabilité des ouvrages. Après plusieurs décennies, l'état global de nombre de ces ouvrages permet d'envisager leur maintien en service ou un changement de fonction moyennant un suivi, une maintenance et des réparations. Le comportement spécifique de ces structures ne peut plus être analysé au travers des schémas standard de conception (hypothèses non valides) et impose le développement de nouveaux modèles intégrant notamment la complexité structurelle, l'évolution des propriétés des matériaux, le critère d'intégrité et une fonction de coût. Elle exprime les impacts économiques d'une ruine à différents niveaux : pertes de vies humaines, conséquences écologiques, conséquence économique directe ou indirecte, réparation... Actuellement, aucun indicateur d'intégrité ne peut servir de base à une analyse structurelle globale. Les méthodes les plus abouties s'appuient sur l'analyse système [LAB 99]. Les schémas de ruine structurelle sont alors discrétisés en événements de ruine locale dont différentes combinaisons conduisent à la ruine de la structure. La transcription graphique de ces analyses est appelée arbre de ruine.

Compte tenu de l'incertitude sur les modèles utilisés et de l'aléa sur les propriétés des matériaux vieillis, une approche probabiliste est en général nécessaire. Elle permet d'intégrer cette réalité tout en explicitant la mesure de la sécurité par rapport à un niveau requis. Dans le secteur pétrolier offshore, il faut ajouter un aléa important sur l'évaluation du chargement marin et une telle approche est incontournable.

Deux analyses complémentaires sont en général menées. L'analyse déterministe ou push-over consiste à incrémenter le chargement extérieur sur une structure jusqu'à la ruine globale. Un seul scénario est déterminé. L'analyse en fiabilité permet quant à elle un encadrement progressif de la fiabilité de la structure au fur et à mesure des scénarios rencontrés. Plusieurs scénarios, correspondant aux probabilités d'occurrence les plus fortes, sont en général prospectés.

Les intercomparaisons menées au cours de la dernière décennie ont montré que les résultats obtenus par l'une et l'autre de ces méthodes étaient fortement dépendants du code de calcul numérique, de l'algorithme du calcul en fiabilité et du processeur. Les hiérarchies des ruines sont quelquefois concordantes mais il est raisonnable de douter du caractère absolu du résultat obtenu pour une structure donnée. La seule alternative est une comparaison avec d'autres structures de même typologie, à partir d'un retour d'expérience.

Cet article se propose de compléter ces deux approches par une analyse de sensibilité de l'arbre de ruine aux hypothèses. Une approche semi-probabiliste permet d'intégrer différentes hypothèses statistiques sur l'état des sections et une incrémentation du chargement fournit un scénario de ruine. La sensibilité du scénario aux hypothèses est étudiée via la création de scénarios parallèles. Pour les 
illustrations, les structures pétrolières offshore de type Jacket serviront de support et on privilégiera les ruines par plastification. La présentation de cette méthode comporte trois volets.

La deuxième partie rappelle le contexte de maintenance d'une structure pétrolière offshore ainsi que les définitions et hypothèses de base pour l'étude de leurs schémas de ruine et poursuit sur les lignes directrices de l'analyse système.

La troisième partie compare des arbres de ruine obtenus par des approches déterministes et en fiabilité et montre l'intérêt de l'approche semi-probabiliste dans le but de fournir à l'opérateur un complément dans le schéma d'aide à la décision et un classement des nœuds critiques.

La quatrième partie propose une représentation globale de la réserve d'intégrité structurelle et une classification de sections critiques associées, complémentaire de la précédente.

\section{L'analyse système : support pour la programmation des plans IMR}

\subsection{Inspection Maintenance Réparation des structures pétrolières}

L'analyse de structures anciennes, en fin de durée d'exploitation certifiée, vise à se prononcer sur la question du prolongement de leur durée de service avec un niveau de réhabilitation à spécifier ou de leur destruction. Ceci suppose le développement de modèles et d'outils spécifiques. Dans le secteur pétrolier offshore, le programme du Club de Liaison pour les Actions de Recherche sur les Ouvrages en Mer intitulé "Requalification des Structures Offshore Existantes » (1994-1998) a abouti au regroupement opérationnel et au développement de ces outils dans l'objectif de pouvoir intégrer de nouvelles données (environnement, matériau et fonctionnalités de la structure) dans un code de calcul opérationnel (logiciel RASOS - programme Brite P-1270, 1991, [RAS 99]). La question est alors de donner des indicateurs mécaniques et fiabilistes permettant à l'opérateur de juger de l'intérêt, au sens d'une fonction coût, du maintien en service de la structure mais aussi des inspections à envisager. Les notions d'intégrité structurelle et de durée de vie résiduelle d'un composant sont au cœur de cette analyse [ROU 9]. Toutes les approches s'appuient sur une évaluation locale du niveau de ruine sous la forme d'un critère d'état limite.

A titre d'illustration, pour des structures métalliques composées de tubes soudés et appelées Jacket (figure 1), le composant de ruine locale est un ensemble comprenant :

- le composant structurel critique (section d'extrémité ou composant entier),

- le critère d'état limite (plastification d'extrémité, flambement, longueur critique de fissure...),

- le comportement post-ruine (plastification avec ou sans écrouissage). 


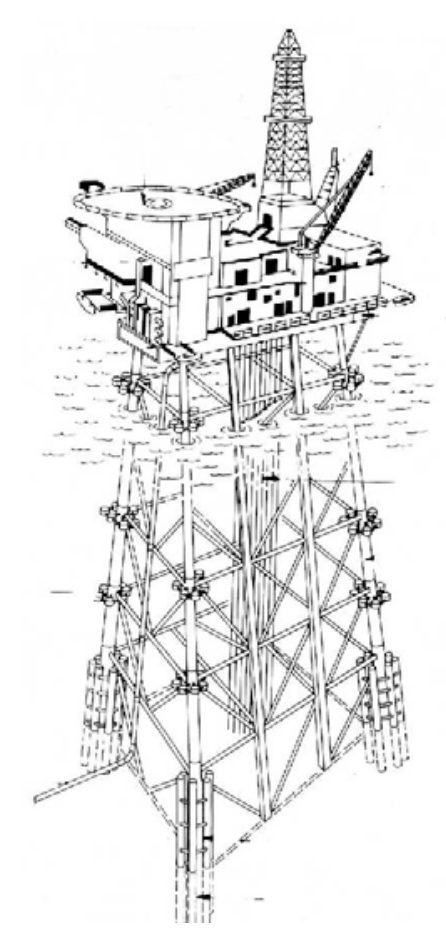

Figure 1. Plate-forme Jacket

Pour une plastification d'extrémité de tube, la fonction d'état limite s'écrit :

$$
g=\frac{M}{M_{p}}-\cos \left(\frac{\pi}{2} \cdot \frac{N}{N_{p}}\right)
$$

sous l'hypothèse d'un comportement élasto-plastique. $M_{P}$ et $N_{P}$ sont respectivement le moment fléchissant plastique et l'effort normal plastique de la section (contrainte limite atteinte en tout point de la section).

\subsection{Analyse système des structures fortement redondantes}

L'analyse système suppose qu'un scénario de ruine globale de la structure est composé d'une succession de ruines locales au sens défini précédemment, chaque élément ruiné à l'étape précédente adoptant ensuite un comportement post-ruine. Trois modes de comportement de la structure sont en général étudiés :

- comportement en fatigue (cycles de houle),

- comportement quasi statique sous chargement extrême (tempêtes),

- comportement en dynamique, ne concernant pas les structures Jacket.

Plusieurs types d'analyse système en découlent :

- analyse déterministe de la structure en conditions de push-over,

- analyse système en fiabilité en conditions extrêmes, 
- analyse système en fiabilité en fatigue,

- analyse système en fiabilité en conditions couplées extrêmes et fatigue.

Seuls des développements récents ([RAS 99], [SCH 99]) permettent une combinaison du chargement en fatigue et du chargement extrême et, en général, une attitude conservative recommande d'étudier la résistance de la structure suivant ces deux comportements de manière distincte. Pour les illustrations, on présentera ici les deux premières analyses qui correspondent à des conditions de tempêtes.

L'analyse déterministe appelée aussi push-over (section 17 de l'American Petroleum Institute : Assessment of Existing Platforms) consiste en un calcul des chargements nodaux dus à un niveau de houle donné, incrémentés par application d'un facteur d'amplification $\lambda$ identique sur toute la structure. Cette approche ne respecte pas la physique de la cinématique de la houle et des alternatives sont en cours de développement. On construit ainsi pas à pas une branche unique représentant les ruines successives de composants structurels.

L'approche en fiabilité propose un autre mode d'identification des scénarios et conduit à la construction d'un arbre à plusieurs branches ayant des probabilités d'occurrence différentes. On se base sur la modélisation probabiliste des variables de base du calcul comme la limite élastique du matériau et les grandeurs caractéristiques de la houle. On recherche d'abord les scénarios de ruine dominants (au sens de leur probabilité d'occurrence et des conséquences de la ruine) intégrant toutes les possibilités de ruines locales. On calcule ensuite leur probabilité d'occurrence à partir de la probabilité d'occurrence de chacune des ruines élémentaires. La probabilité de ruine système est alors encadrée par des probabilités de ruine calculées comme une combinaison d'unions et d'intersections de ces scénarios. Notons que la probabilité de ruine peut être parfaitement encadrée tout en étant biaisée par l'oubli d'un scénario dominant. L'arbre de ruine récapitule les branches correspondant à ces scénarios, chaque nœud correspondant à une ruine élémentaire (numéro de l'élément et type d'état limite) dont on reporte l'indice de fiabilité à cette étape de calcul. Compte tenu du volume de calcul envisagé, des approches simplifiées sont en général proposées dans les codes de calcul de type RASOS.

Les résultats obtenus par ces approches sont très sensibles au code de calcul utilisé et aux algorithmes d'optimisation. Ils n'ont donc aucune valeur absolue mais permettent toutefois de réaliser une hiérarchie des scénarios au sein de la structure. Le danger majeur est d'oublier une partie terminale d'un scénario dominant et de minorer les deux bornes d'encadrement de la probabilité de ruine. Une troisième approche, dite de sensibilité, sera proposée ensuite et est particulièrement adaptée au cas des structures existantes. 


\section{Analyses déterministes, fiabilistes et approche semi-probabiliste}

\subsection{Présentation du cas d'étude et des résultats par les approches traditionnelles}

On étudie une structure offshore de $56 \mathrm{~m}$ de haut avec des éléments tubulaires en acier de diamètre extérieur allant de $30 \mathrm{~cm}$ à $1,2 \mathrm{~m}$ pour les jambes. Pour faciliter les comparaisons, seul l'état limite de ruine par plastification d'extrémité est considéré. La géométrie et le mode de chargement de la houle sont schématisés en figure 2. Cette structure est référencée précisément dans la littérature [MOH 93]. L'arbre de ruine en fiabilité est présenté en figure 3 [ $\mathrm{MOH}$ 93]. Les indices de fiabilité reportés dans les cercles du diagramme sont des indices $\beta$ au sens d'Hasofer-Lind [HAS 74]. La probabilité de ruine $\mathrm{P}_{\mathrm{r}}$ varie dans le sens inverse de l'indice $\beta\left(\beta=0 \Rightarrow \mathrm{P}_{\mathrm{r}} \approx 50 \%\right)$.

La branche correspondant au scénario unique obtenu par l'analyse de type pushover est présentée en figure 4, toujours au sens de l'état limite de plastification d'extrémité. La section est dite plastifiée lorsque la fonction d'état limite $\mathrm{g}$, à valeurs dans $[0 ; 1]$, prend une valeur inférieure ou égale à 0,01 . Le comportement post ruine comporte un léger écrouissage pour parer à des problèmes numériques.

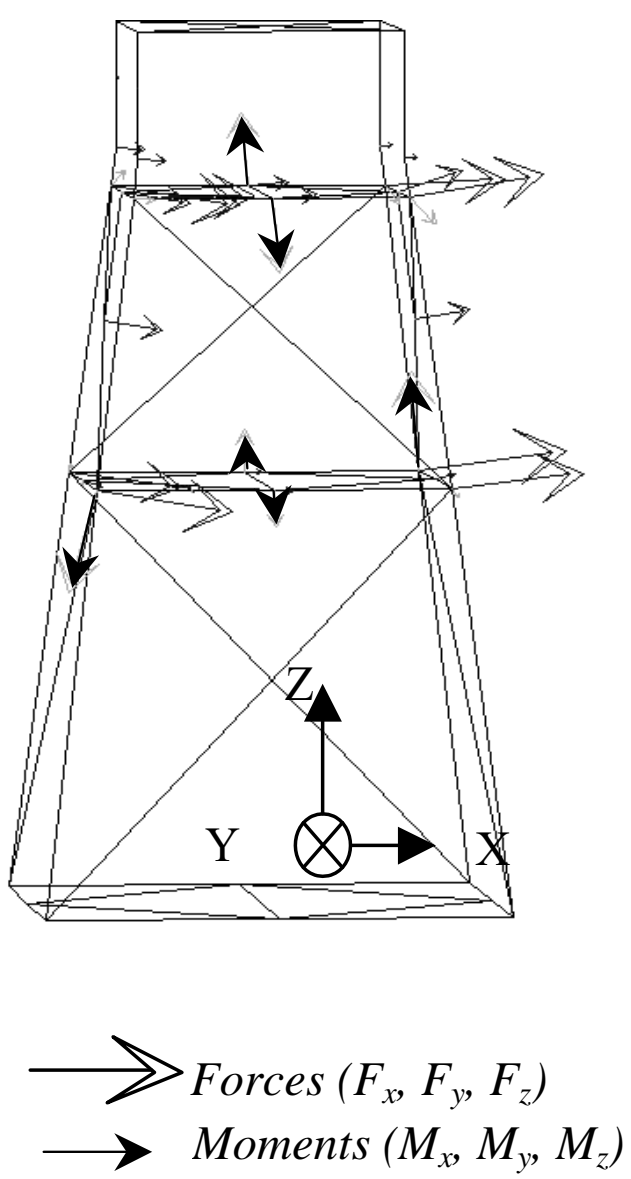

Figure 2. Schéma simplifié: plate-forme et chargement 
$S_{63}^{32}$ : rotule platique

élément $n^{\circ} 63$, section 32

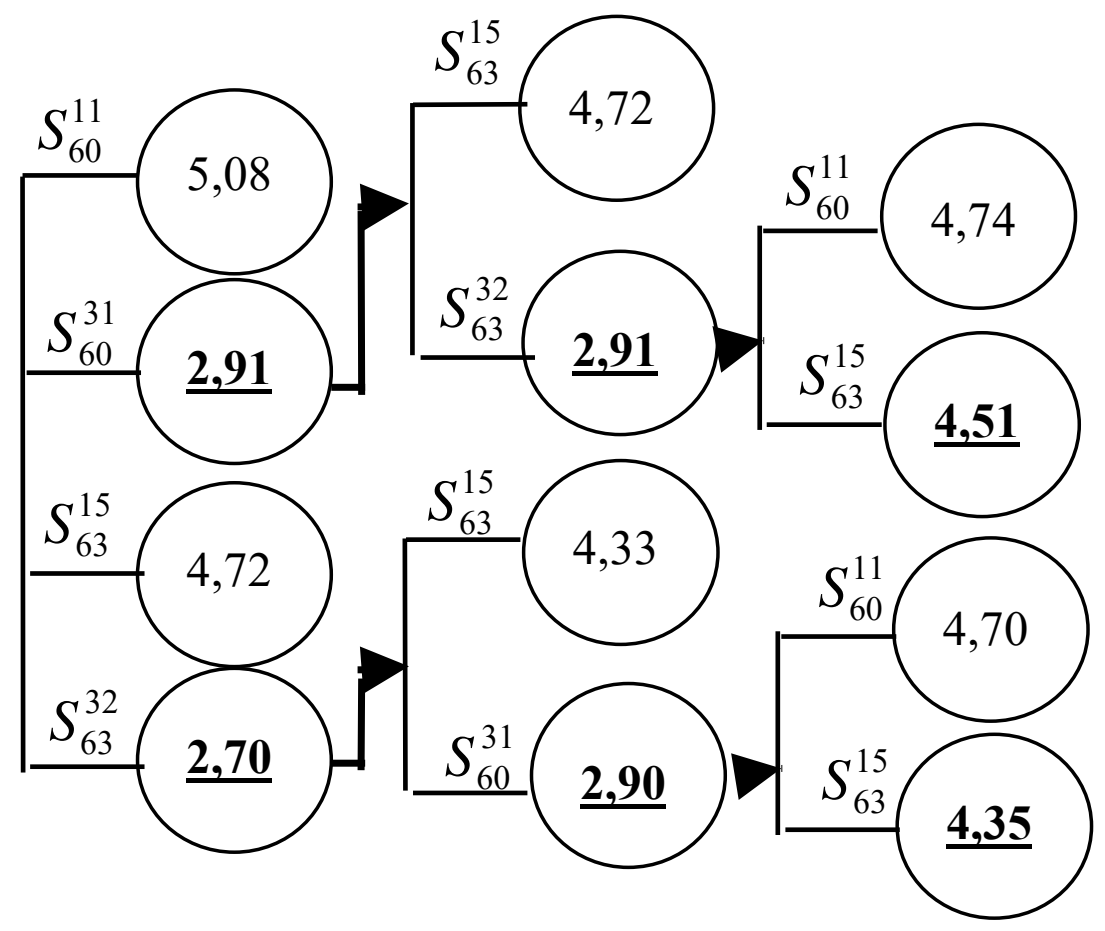

Figure 3. Arbre de ruine en fiabilité

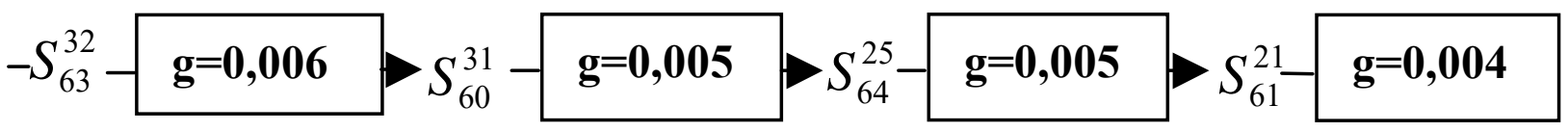

Figure 4. Branche de l'arbre de ruine par l'analyse déterministe de type push-over

\subsection{Approche semi-probabiliste de la sensibilité d'un arbre de ruine}

Comme énoncé précédemment, une source majeure d'incertitude lors de la réanalyse de structures existantes réside dans les propriétés du matériau. La sensibilité des scénarios de ruine est quant à elle étroitement liée à ces caractéristiques. C'est pourquoi on présente ici une étude de sensibilité centrée sur cette source d'incertitude. La structure est envisagée dans son comportement en conditions de tempête, le mode de ruine local considéré étant restreint, comme au paragraphe 3.1, à la plastification d'extrémité et l'intégrité structurelle, considérée au sens de la marge de sécurité, est calculée avec la fonction d'état limite par plastification g. Cette méthode est à rapprocher d'une analyse de type push-over avec une étude de sensibilité sur la valeur de la résistance caractéristique limite $f_{k}$ relative au quantile de $\mathrm{k} \%$ en supposant une distribution lognormale de cette variable. On étudie ainsi la sensibilité de la caractéristique d'un élément par l'impact de son augmentation en valeur caractéristique sur l'intégrité de la structure. Lors d'un premier calcul incrémental, on évalue la suite des sections plastifiées afin d'obtenir une branche du scénario de ruine. On augmente la résistance $f_{y}$ de la première section de cette branche et on recommence la simulation incrémentale afin d'obtenir une seconde branche. On recommence ainsi de suite afin d'explorer plusieurs branches de l'arbre 
de ruine. Cette approche est synthétisée sur l'algorithme présenté figure 5 et l'arbre de ruine partiel correspondant figure 6. Un arbre plus complet est disponible dans [SCH 99].

Une analyse comparative avec l'approche en fiabilité permet de confirmer deux éléments fondamentaux : les scénarios obtenus par cette dernière sont dominants et ont un sens mécanique. On obtient les mêmes sections critiques pour les deux approches (numéros de sections soulignés en figure 6). Cependant, l'approche semiprobabiliste fait émerger deux sections absentes de l'analyse en fiabilité $\left(S_{64}^{25}\right.$ intercalée dans un scénario de la première branche et $S_{61}^{21}$ dont le rôle fondamental apparaît dans chaque branche). Ces informations permettent d'orienter l'étude en fiabilité vers ces branches afin de vérifier si tout ou partie de ce scénario est dominant au sens de la ruine structurelle.

On peut de plus envisager un classement des sections sur critère local en trois classes de criticité au sens de cette construction d'arbre de ruine.

\begin{tabular}{|l|l|l|}
\hline Classe 1 & $\begin{array}{l}\text { Sections dominantes (premiers nœuds ou nœuds présents dans } \\
\text { plusieurs branches) }\end{array}$ & $\begin{array}{l}\text { Sections } \\
\mathbf{3 2 , \mathbf { 3 1 }}\end{array}$ \\
\hline Classe 2 & $\begin{array}{l}\text { Sections critiques (nœuds de la première branche) non } \\
\text { présents dans la classe 1 }\end{array}$ & $\begin{array}{l}\text { Sections } \\
\mathbf{2 5 , 1 5}\end{array}$ \\
\hline Classe 3 & $\begin{array}{l}\text { Sections sensibles (nœuds de la seconde branche) non présents } \\
\text { dans les classes 1 et 2 }\end{array}$ & $\begin{array}{l}\text { Section } \\
\mathbf{2 1}\end{array}$ \\
\hline
\end{tabular}

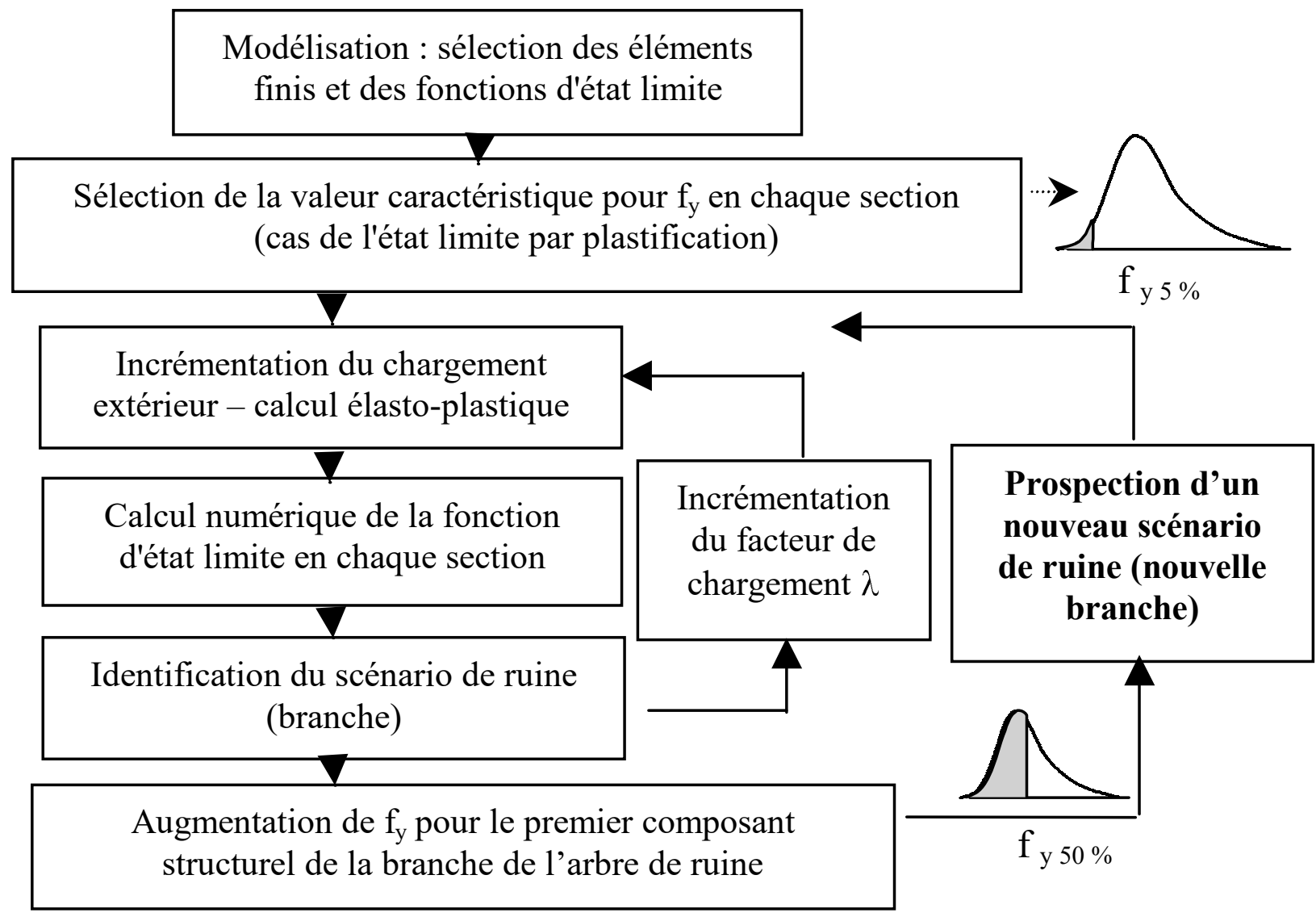

Figure 5. Algorithme de prospection d'un arbre de ruine par analyse de sensibilité 


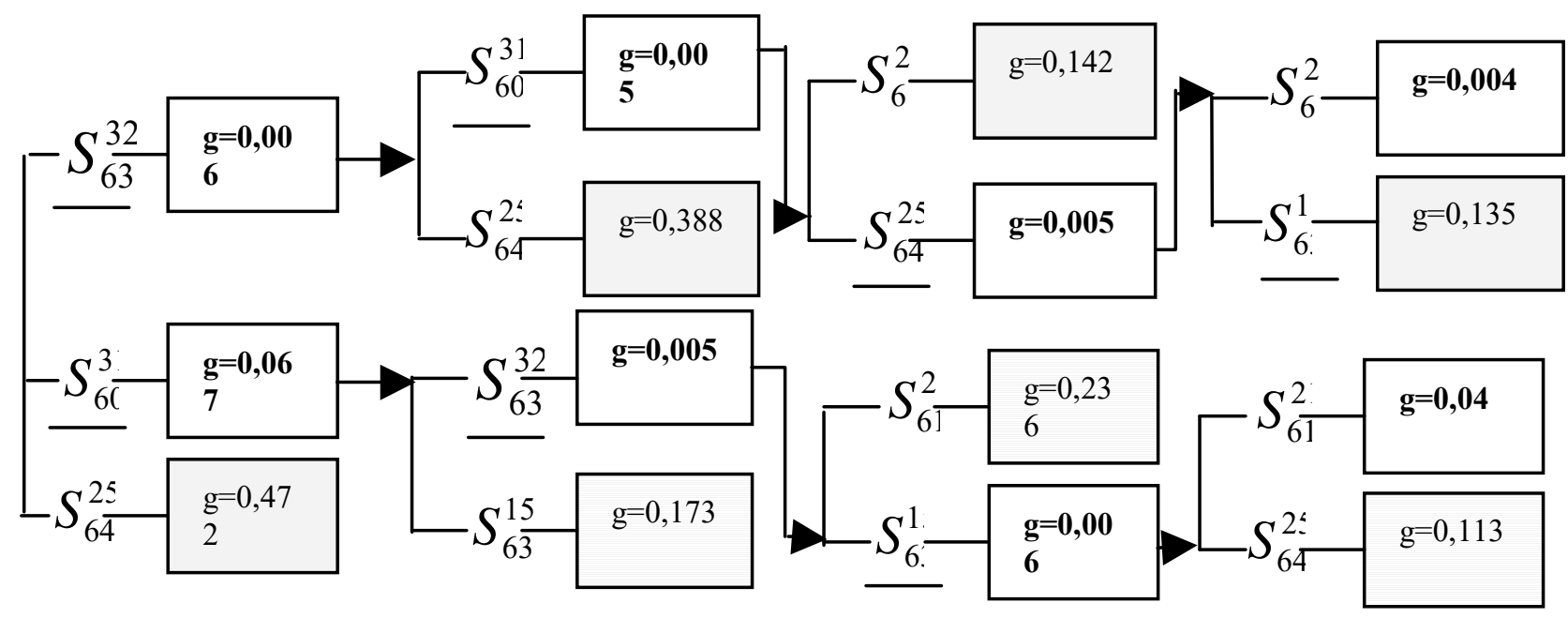

Figure 6. Arbre de ruine partiel obtenu par l'approche semi-probabiliste

\section{Critère déterministe global pour le classement de sections critiques}

L'approche semi-probabiliste présentée dans la section précédente peut être considérée comme complémentaire des analyses en fiabilité système ou push-over. Ces méthodes sont basées sur une classification par critère d'intégrité locale. L'intégrité de la structure ne peut être que partiellement appréhendée. On propose ci-dessous un critère global comme critère rationnel d'aide à la décision pour la compréhension de la réserve d'intégrité d'une structure. En notant $g_{i}(\lambda)$ la valeur de la fonction d'état limite en section i pour le facteur de chargement $\lambda$ sous un état limite spécifique, la réserve d'intégrité globale de la structure au sens de cet état limite peut être interprétée par le suivi d'évolution de l'indice de défaillance global $\mathrm{G}$ en fonction de $\lambda$ :

$$
G(\lambda)=\frac{\sum_{i=1}^{N} g_{i}(\lambda)}{N}
$$

où $\mathrm{N}$ est le nombre total de sections de la structure. Toutes les sections ont ainsi le même poids pour la calcul de l'indice global G. Les variations importantes de G sont représentatives d'un ensemble de défaillances se produisant à niveau de facteur de chargement donné.

La figure 7 représente les courbes $\mathrm{g}_{\mathrm{i}}$ et l'évolution de $\mathrm{G}$ avec $\lambda$ pour la structure présentée en section 3. Les calculs sont réalisés sous CAST3M avec comportement élasto-plastique et écrouissage très faible assurant la convergence.

Pour les configurations étudiées ici, $\mathrm{N}$ est grand et la fonction $\mathrm{G}$ peut être considérée comme continue. Elle est par ailleurs positive, décroissante et à valeurs dans [0;1], 0 étant valeur asymptotique. Il existe par conséquent un ou plusieurs points d'inflexions, représentatifs d'une dégradation importante au voisinage d'un 
facteur de chargement. On détecte ces « ruptures » par franchissement de l'axe des abscisses de la fonction $\left(\delta^{2} \mathrm{G} / \delta \lambda^{2}\right)$ pour les points où $(\delta \mathrm{G} / \delta \lambda)$ est minimum [SCH 99]. Pour l'exploitation des résultats, on suppose qu'il y a ruine lorsque g est inférieure à 0,005 . Ces résultats font ressortir deux classes de sections, reflétant deux types d'inflexion de $\mathrm{G}$ donc deux niveaux de scénarios critiques pour la structure.

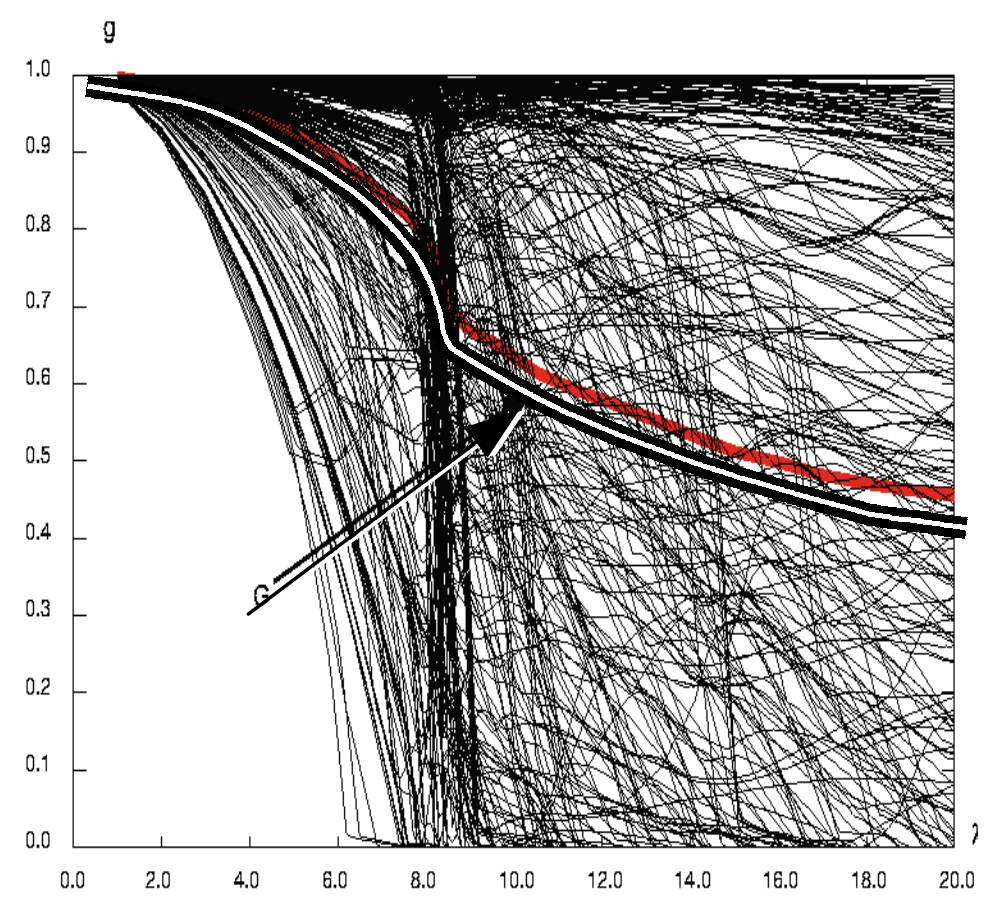

Figure 7. Evolution de $G$ et des fonctions $g_{i}$ en fonction de $\lambda$

\begin{tabular}{|l|l|l|}
\hline Classe I & Inflexion importante de G & 25 sections concernées $(\lambda=\mathbf{8 , 3})$ \\
\hline Classe II & Faible inflexion de G & 4 sections $S_{63}^{15} S_{63}^{32} S_{64}^{25} S_{60}^{31}(\lambda=\mathbf{7 , 1})$ \\
\hline
\end{tabular}

L'étude de cet indice global de défaillance $\mathrm{G}$ donne des informations pertinentes sur le comportement local et global de la structure. Toutefois la méthode choisie pour son calcul ne prend pas en compte le caractère plus ou moins critique des sections. Notamment, on peut envisager que le calcul d'une structure ayant beaucoup d'éléments dans une zone non sollicitée ne donnera pas le même résultat que celui ayant peu d'éléments dans cette zone. On peut envisager d'affiner le calcul de $\mathrm{G}$ en lui associant l'énergie résiduelle de la structure. Il faut noter l'importance de relier ces méthodes au code de calcul et contraintes technologiques ayant présidé au dimensionnement de la structure (contexte réglementaire initial). 


\section{Conclusion}

L'analyse de structures anciennes, en fin de durée d'exploitation réglementaire, nécessite de reposer les décisions d'Inspection Maintenance Réparation sur des critères rationnels d'aide à la décision. Une analyse système de sensibilité apparaît comme complémentaire aux résultats donnés par les arbres de ruine obtenus en fiabilité. Elle permet de donner une interprétation plus mécanique de ces résultats en concentrant l'aléa sur les caractéristiques des matériaux, fondamentales pour ce type de structures, le chargement et le modèle mécanique étant déterministes. Elle peut à ce titre orienter des compléments d'études de fiabilité qui auraient négligé certains scénarios de ruine. On peut en déduire une classification de la criticité des sections sur critère local et on montre qu'un critère global peut constituer une source complémentaire d'information afin d'évaluer la nocivité d'un scénario sur la perte d'intégrité structurelle.

\section{Bibliographie}

[GOY 96] GoYet J., Maroini A., «Offshore Platform Reliability - Optimal inspection and repair planning: an application with a sensitivity study using IMREL Methodology », Proc. Internat. Conf. on fatigue of welded components and structures, Soc. Fran. de Métallurgie et de Matériaux - Fatigue Commission, Senlis, juin 1996.

[GUE 94] GUENARD Y.F., Application of system reliability analysis to offshore structures, Report $\mathrm{n}^{\circ}$ 71, The John A. Blume Earthquake Engineering Center, Nov. 1994, p. 1-36, 129-149.

[HAS 74] HASOFER A.M., LiND N.C., "Exact and invariant second moment code format », Journal of Engineering Mechanics, vol. 100, p. 111-121, 1974.

[LAB 99] Labeyrie J., Goyet J., Schoefs F., « Une Approche Probabiliste Système pour la Requalification des Plate-formes Pétrolières », Seconde conf. nat., JN-FIAB'98, Marnela-Vallée, Nov. 1998, «Fiabilité des Matériaux et des Structures », Part.1 Fiabilité et réglementation, Hermès, 1999, p. 39-48.

[MAR 93] Aroini A., Birades M., Goyet J., Prevosto M., « A reliability based methodology of requalification for existing jacket platforms », Proc. 12 th Int. Conference on Offshore Mechanics and Arctic Engineering, vol. 2, ASME New York 1993, p. 335-349.

[MOH 93] MOHAMED A., Modèle mécanofiabiliste linéarisé pour l'analyse des structures Application aux plates-formes marines, Mémoire de thèse de doctorat, Univ. de Clermont-Ferrand, 1993.

[RAS 99] RASOS, Version 6, User's manual, Atkins report, 1999.

[ROU 99] Rouhan A., Schoefs F., «On the use of inspections results in IMR plans», Second International Conference on Decision Making in Urban and Civil Engineering, Lyon, 20-22 novembre, vol. 2, p. 1259-1270, 2000.

[SCH 99] Schoefs F., Dube J.F., Goyet J., «Semiprobabilistic analysis and instability detection on failure trees as rational aid tools for existaing structures reassessment ", Second International Conference on Decision Making in Urban and Civil Engineering, Lyon, 20-22 novembre, vol. 2, p. 857-868, 2000. 